\title{
Objective Fall Risk Detection in Stroke Survivors Using Wearable Sensor Technology: A
}

\section{Feasibility Study}

Running Head: Fall Risk Detection in Stroke Survivors

Authors: Ruth E. Taylor-Piliae, PhD, RN, FAHA ${ }^{1}$; M. Jane Mohler, PhD, MPH, NP ${ }^{1,2}$; Bijan Najafi, $\mathrm{PhD}^{2}$; and Bruce M. Coull, $\mathrm{MD}^{2}$

Affiliations: ${ }^{1}$ College of Nursing, University of Arizona, Tucson, AZ; ${ }^{2}$ College of Medicine, University of Arizona, Tucson, AZ

Address for Correspondence: Dr. Ruth E. Taylor-Piliae, College of Nursing, University of Arizona, 1305 N. Martin, PO Box 210203, Tucson, AZ 85721-0203 (tel: 520-626-4881; fax: 520-626-4062;

email: rtaylor@nursing.arizona.edu)

Word count: 2,002

Tables: 3

\section{Figures: 1}

Conflict of Interest: The authors have no conflict of interest to report.

Financial Support/Disclosures: This study was funded by an American Heart Association (AHA) Scientist Development Grant (\#0930324N; Taylor-Piliae, PI) and a University of Arizona, Laurence B. Emmons Research Grant (\#5330000-EMMRT; Taylor-Piliae, PI). The funding sources did not participate in the study design, in the collection, analysis, and interpretation of data; in the writing of the report; or in the decision to submit the article for publication. Preliminary results from this study were presented at the American Heart Association/American Stroke Association's International Stroke Conference, San Diego, CA; February 11-14, 2014. 
Acknowledgements: The authors would like to thank all of the study participants and our dedicated study staff: Daniella Boros, BS; and Gurtej Grewal, PhD for their help with recruitment and data collection.

Adherence to Ethics/Reporting Requirements: Normal ethical procedures were adhered to when conducting this research. 

Feasibility Study

3 Abstract

4 Background: Stroke survivors often have persistent neural deficits related to motor function

5 and sensation, which increases their risk of falling, most of which occurs at home or in

6 community settings. The use of wearable technology to monitor fall risk and gait in stroke

7 survivors may prove useful in enhancing recovery and/or preventing injuries.

8 Objective: Determine the feasibility of using wearable technology (PAMSys ${ }^{\mathrm{TM}}$ ) to objectively

9 monitor fall risk and gait in home and community settings in stroke survivors.

10 Methods: In this feasibility study, we used the PAMSys ${ }^{\mathrm{TM}}$ to identify fall risk indicators (postural

11 transitions: duration in seconds, and number of unsuccessful attempts), and gait (steps, speed,

12 duration) for 48 hours during usual daily activities in stroke survivors ( $n=10)$, compared to age-

13 matched controls $(n=10)$. A questionnaire assessed device acceptability.

14 Results: Stroke survivors mean age was $70 \pm 8$ years old, were mainly Caucasian (60\%) women

15 (70\%), and not significantly different than the age-matched controls (all $p$-values $>0.20$ ). Stroke

16 survivors (100\%) reported that the device was comfortable to wear, didn't interfere with

17 everyday activities, and were willing to wear it for another 48 hours. None reported any

18 difficulty with the device while sleeping, removing/putting back on for showering or changing

19 clothes. When compared to controls, stroke survivors had significantly worse fall risk indicators 20 and walked less $(p<0.05)$

21 Conclusion: Stroke survivors reported high acceptability of 48 hours of continuous PAMSys ${ }^{\mathrm{TM}}$ 
22 monitoring. The use of in-home wearable technology may prove useful in monitoring fall risk

23 and gait in stroke survivors, potentially enhancing recovery.

24 Keywords: accidental fall risk; ambulatory monitoring; feasibility study; gait 


\section{Objective Fall Risk Detection in Stroke Survivors Using Wearable Sensor Technology: A}

\section{Feasibility Study}

Introduction

Nearly 800,000 people sustain a stroke in the United States each year. ${ }^{1}$ Stroke survivors often have lingering neurological deficits related to sensorimotor function, which can increase their risk of falling. ${ }^{2,3}$ Community-dwelling stroke survivors fall up to seven times more annually, than healthy adults of a similar age. ${ }^{4}$ Stroke survivors who fall, often fall repeatedly. ${ }^{4}$ Falling after a stroke often results in major personal and public health losses due to hip or other fractures, loss of independence, fear of falling, activity restriction, reduced mobility and social isolation. ${ }^{4-6}$ Since fall-related injuries represent an enormous and growing burden to all stroke survivors, their families, society, and to our health care system (direct medical costs $\$ 28.3$ billion); preventing falls and fall-related injuries is critical. ${ }^{7,8}$ The risk of falling is a persistent and serious concern regardless of the length of time post-stroke. ${ }^{4}$

Clinical and laboratory-based measures (e.g., timed up and go test, balance tests) have failed to predict falls or fall risk with substantial sensitivity $\cdot{ }^{9-11}$ Moreover, many of the existing screening tools (e.g., timed up and go test) tend to discriminate poorly between fallers and non-fallers. ${ }^{12}$ An innovative, light weight, wearable, mobile, kinematic motion sensor (i.e., PAMSys $^{\mathrm{TM}}$ ) for objectively monitoring fall risk (postural transition), and gait (steps, speed, duration) in naturalistic environments (i.e., home and community) has recently been developed. ${ }^{13-15}$ Validating the use of a wearable mobile technology for objectively monitoring fall risk and gait in naturalistic environments among stroke survivors is important, yet lacking in the current scientific body of knowledge. 
The goal of this study was to determine the feasibility of using a kinematic motion sensor (PAMSys ${ }^{\mathrm{TM}}$, Biosensics LLC, MA, USA) to objectively monitor fall risk and gait in naturalistic environments in community-dwelling stroke survivors. The specific aims were to: 1.$)$ Determine the acceptability of wearing the PAMSys ${ }^{\mathrm{TM}}$ Equipment (i.e., t-shirt with velcro pocket

27 closure for sensor) for 48 consecutive hours ascertaining both comfort and challenges; 2 .) Determine fall risk indicators (postural transition) and gait parameters (steps, speed, duration); and 3.) Compare fall risk indicators and gait data obtained using the PAMSys ${ }^{\mathrm{TM}}$, with data from age-matched controls collected in a prior study among a non-frail population cohort (Clinical Trials.gov, Identifier \#NCT01880229).

\section{Methods}

Design

This feasibility study used a prospective, observational approach.

\section{Participants}

Community-dwelling stroke survivors from all sex/gender and racial/ethnic groups, aged $\geq 50$ years, at least 3 months post-stroke, and living in the Tucson, AZ area were targeted for study enrollment. Stroke survivors that walked with an assistive device (e.g., cane or walker) were eligible. Potential participants were recruited from multiple sources, including a list of prior study participants that agreed to be contacted for future studies, outpatient stroke rehabilitation centers, senior centers, and neurology/neurosurgery offices. For safety reasons, those not eligible for participation included stroke survivors who had a serious medical condition that would interfere with study participation (e.g., myocardial infarction or cardiac surgery in the past 3 months or active treatment for cancer). Approval to conduct the study was 
45

obtained from the Institutional Review Board at the University of Arizona. The investigation was carried out according to the principles outlined in the Declaration of Helsinki, including written informed consent from all subjects. For this feasibility study, we enrolled 10 communitydwelling stroke survivors. ${ }^{16}$

Procedures

All interested stroke survivors were interviewed either in person or by telephone to determine eligibility using a self-report screening questionnaire. If the study eligibility criteria were met, participants were scheduled at a convenient time for a one-hour study appointment, upon which written informed consent was obtained, prior to any data collection. Data were collected in the Interdisciplinary Consortium for Advanced Motion Performance (iCAMP) Laboratory at the University of Arizona. All participants received a map to iCAMP with parking directions.

Self-reported demographics (e.g., age, gender) and stroke history (e.g., stroke type, months post-stroke) were collected from each participant at the study appointment. The participants were instructed on the use of the PAMSys ${ }^{\mathrm{TM}}$, which they wore for 48 consecutive hours and then mailed back in a laboratory-addressed, pre-paid Fed-Ex envelope. Participants were telephoned on the third day following the study visit to determine the acceptability of the PAMSys $^{\mathrm{TM}}$ and assure return of the PAMSys ${ }^{\mathrm{TM}}$ Equipment. To ascertain PAMSys ${ }^{\mathrm{TM}}$ Equipment acceptability (e.g., comfort, challenges), study staff interviewed participants using an investigator-developed questionnaire (14 yes/no questions, see Appendix). Questions related to comfort of the PAMSys ${ }^{\mathrm{TM}}$ included: "Did you wear the PAMSys ${ }^{\mathrm{TM}}$ equipment (t-shirt and sensor) for the full 48 hours?"; "Would you be willing to wear the PAMSys ${ }^{\mathrm{TM}}$ equipment (t-shirt 
67 and sensor) for another 48 hours?". Questions related to potential challenges included: “Did 68 you have any difficulty removing the PAMSys ${ }^{\mathrm{TM}}$ to take a shower?"; "Did you have trouble 69 sleeping while wearing the PAMSys ${ }^{\mathrm{TM}}$ equipment?" In addition, we monitored actual falls and 70 adverse events reported by participants during the study (48 consecutive hours of wearing

71 PAMSys ${ }^{\mathrm{TM}}$ Equipment). Upon complete data collection, the participant was thanked and a small

72 incentive (\$25 gift card) to cover the costs of participation was mailed to them.

73 Kinematic Motion Sensor: PAMSys ${ }^{T M}$

PAMSys $^{\mathrm{TM}}$ is a small $(5.1 \times 3 \times 1.6 \mathrm{~cm})$, light $(24 \mathrm{~g})$, wearable sensor technology containing a

75 tri-axial accelerometer. The system computes various parameters associated with the

76 participant's movement from the data recorded by the PAMSys ${ }^{\mathrm{TM}}$ unit placed in a mid-sternal

77 pocket located in a comfortable t-shirt (see Figure 1). The PAMSys ${ }^{\mathrm{TM}}$ technology uses validated

78 advanced signal processing algorithms and biomechanical models of human motion to identify

79 posture (walking, sitting, standing, lying), postural transitions (fall risk indicators: duration in

80 seconds, and number of unsuccessful attempts) and gait (steps, speed, duration). ${ }^{14,15}$ These

81 parameters consist of: (a) the participant's trunk tilt (specified in degrees, measuring the angle

82 between the participant's trunk axis, and the axis aligned with the gravitational force); (b) the

83 type of the participant's postural transitions (e.g., sit-to-stand); (c) duration of the participant's

84 postural transitions; (d) duration of the participant's locomotion (number and duration of

85 continuous walking bouts); (f) characterization of the participant's locomotion (gait speed and

86 number of steps); and (g) type of the participant's postures (walking, sitting, standing, lying).

87 The details of the algorithms to identify each posture has been described and validated 
89 subsequently to classify the transitions between postures, i.e., sitting-to-standing, standing-tositting, lying-to-sitting, and sitting-to-lying; for determining fall risk. ${ }^{14,15,18,19}$ Lying posture is

91 identified by measuring the trunk angle from the accelerometer data. Postural transition and

92 duration are identified by measuring the pattern recognition of the trunk tilt. Then postural transition is classified as sitting or standing via pattern recognition of the vertical

94 accelerometer. The PAMSys ${ }^{\mathrm{TM}}$ can quantify postural transition including number of successive 95 postural transitions (unsuccessful attempts rising from a chair), number and duration of postural transitions, and postural transition variability. These parameters were able to

97 distinguish between older adults at low and high risk of falling. ${ }^{14}$ Gait parameters include the 98 number of steps, walking speed, and amount of walking. ${ }^{18}$ Gait speed and number of steps are detected according to the vertical accelerometer peaks satisfying pre-defined conditions. Gait speed is detected by measuring step time and intensity of the vertical and frontal

101 accelerometers. Finally, a series of rules are applied to enhance accuracy and identify 102 misclassification errors (e.g., walking during sitting is unlikely; leaning backward for extended 103 period of time during standing is unlikely). The PAMSys ${ }^{\mathrm{TM}}$ has an embedded battery that allows 104 data recording in the memory unit (2GB) with a suitable sample-rate frequency $(50 \mathrm{~Hz})$, for up 105 to six days of continuous measurement. The data can be transferred to a computer via a USB 106 reader for analysis. The validity of PAMSys ${ }^{\text {TM }}$ has been demonstrated in four separate pilot studies among 108 both community dwelling and hospitalized older adults ( $n=66$, mean age $=75$ years), by 109 benchmarking the results with an independent analysis using an optical motion system 110 (sensitivity=99\% for postural transition duration; sensitivity/specificity $\geq 87 \%$ for change in 
111 postural transition). ${ }^{15,18}$ Such detection of postural transitions represents a tremendous

112 technologic leap over what is currently available to objectively monitor fall risk, and gait in

113 stroke survivors in naturalistic environments during everyday life.

114 Data Analysis

115 To determine the acceptability of wearing the PAMSys ${ }^{\mathrm{TM}}$ Equipment (i.e., $\mathrm{t}$-shirt and 116 sensor) for 48 consecutive hours, we used descriptive statistics to assess both comfort and 117 challenges. The PAMSys ${ }^{\mathrm{TM}}$ data is complex and provides time stamps for each participant's data.

118 The data were extracted and then analyzed using IBM ${ }^{\circledR}$ SPSS $^{\circledR}$ for Windows, Version 20.0 (IBM

119 Corp). To determine fall risk indicators (postural transition), and gait (steps, speed, duration)

120 among stroke survivors using the PAMSys ${ }^{\mathrm{TM}}$ we used descriptive statistics. We used

121 independent t-tests, to compare fall risk and gait parameters with PAMSys ${ }^{\mathrm{TM}}$ data from age-

122 matched controls collected in a prior study.

\section{Results}

Stroke survivors were on average 70 years old and 3.5 years post-stroke. The majority

125 were retired, college-educated, Caucasian women, reporting an ischemic stroke with

126 hemiparesis. The majority of the age-matched controls were Caucasian women, similar to the

127 stroke survivors. There were no significant differences between the stroke survivors and

128 controls participants according to age, ethnicity, or gender (Table 1).

Stroke survivors (100\%) reported that the PAMSys ${ }^{\mathrm{TM}}$ was comfortable to wear for the full

13048 hours, did not interfere with normal everyday activities and were willing to wear it for

131 another 48 hours. A total of 6 stroke survivors reported removing the PAMSys ${ }^{\mathrm{TM}}$ to take a

132 shower (mean time off=31 minutes); while 4 stroke survivors reported removing the PAMSys ${ }^{\mathrm{TM}}$ 
133 to change clothes (mean time off=11 minutes). None reported any difficulty with the PAMSys ${ }^{\mathrm{TM}}$

134 while sleeping, removing or putting back on for showering or changing clothes or that it

135 became wet or dirty (Table 2). When these stroke survivors were asked what they would like to

136 tell the next participant to expect and share their experience, all (100\%) commented that the

137 PAMSys ${ }^{\mathrm{TM}}$ was comfortable, lightweight, enjoyable, and easy to wear. There were no falls or

138 adverse events reported during the study (48 consecutive hours of wearing PAMSys ${ }^{\mathrm{TM}}$ ).

139 Compared to controls, stroke survivors had significantly worse fall risk indicators $(p<0.05)$,

140 needing on average more than 4 seconds to change their posture (e.g., sit-to-stand) and had on

141 average over 10 failed sit-to-stand attempts per day. In addition, compared to controls, stroke

142 survivors took fewer steps (on average 4337 steps/day) and spent less time walking $(p<0.05)$

143 during the 48-hours of continuous monitoring (Table 3).

144 Discussion

$145 \quad$ Few studies have evaluated body-worn sensors among older adults in home and

146 community settings. ${ }^{20,21}$ To our knowledge, this is the first study conducted in community-

147 dwelling stroke survivors, which has objectively monitored fall risk indicators and gait in home

148 and community settings using a kinematic motion sensor (PAMSys ${ }^{\mathrm{TM}}$ ).

149 One of the issues surrounding the acceptability of body-worn sensors among older

150 adults, reported in the literature, is the potential discomfort of wearing the device for a

151 prolonged period of time ( $>24$ hours). ${ }^{22}$ In our feasibility study, all stroke survivors reported

152 that the PAMSys ${ }^{\mathrm{TM}}$ was comfortable to wear for the full 48 hours, did not interfere with normal

153 everyday activities and were willing to wear it for another 48 hours. None of these stroke

154 survivors reported any difficulty with the PAMSys ${ }^{\mathrm{TM}}$ while sleeping, removing/putting back on 
155 for showering/changing clothes or that it became wet/dirty. Results from our study are similar 156 to findings reported by Bloch and colleagues ${ }^{23}$ examining the acceptability of wearing a body157 worn sensor for an extended period of time ( $>24$ hours) among older adults. Thus, objective 158 cross-sectional monitoring using this kinematic motion sensor is feasible and acceptable.

In this feasibility study, when compared to age-matched controls, stroke survivors had

160 significantly worse fall risk indicators and walked less. These findings are consistent with

161 findings reported by others, ${ }^{24-27}$ that stroke survivors are relatively sedentary when compared 162 to same-aged adults with/without a chronic disease. Our results further highlight the utility of 163 using objective kinematic motion sensors to monitor fall risk and gait in community-dwelling 164 stroke survivors - so that strategies can be implemented early on, to reduce the risk of falling in 165 this vulnerable population. For example, one underutilized strategy is for healthcare providers 166 to provide counseling on increasing physical activity participation and/or recommending a 167 variety of suitable exercise programs, such as Tai Chi or SilverSneakers ${ }^{\circledR}$, to reduce the risk of 168 falling. ${ }^{28-30}$

169 Study Limitations As with any feasibility study, the small sample limits generalizability of the results. We

171 monitored fall risk indicators and gait parameters for 48 hours only, so our results may not be 172 representative of other stroke survivors. However, these stroke survivors reported their 173 willingness to wear the PAMSys ${ }^{\mathrm{TM}}$ for another 48 hours indicating acceptability, there were no 174 reported challenges (e.g., no trouble sleeping with device on) indicating successful 175 implementation, with ease of use (e.g., able to remove and put back on after showering) 176 indicating practicality. 


\section{Conclusion}

178 Falls and fall risk are major concerns for stroke survivors. Since the majority of fall-

179 related events occur at home or in community settings, the use of wearable mobile technology

180 in stroke survivors may be useful to monitor fall risk and gait in these settings, which could

181 further enhance recovery and/or prevent injuries. We have demonstrated the utility of the

$182 \quad$ PAMSys $^{\mathrm{TM}}$ system for incorporation into cross-sectional monitoring of fall risk and gait

183 assessment, with the potential for rehabilitation outcome assessment. As sensor algorithms

184 become increasingly more predictive with less obtrusive applications, the potential for

185 continuous monitoring of those at high risk for falling will develop into practical solutions for

186 home and community settings. 


\section{References}

189 1. Go AS, Mozaffarian D, Roger VL, et al. Heart disease and stroke statistics--2014 update: a report from the American Heart Association. Circulation. 2014;129(3):e28-e292.

2. Patterson SL, Forrester LW, Rodgers MM, et al. Determinants of walking function after stroke: differences by deficit severity. Arch Phys Med Rehabil. 2007;88(1):115-119.

3. Yates JS, Lai SM, Duncan PW, Studenski S. Falls in community-dwelling stroke survivors: an accumulated impairments model. J Rehabil Res Dev. 2002;39(3):385-394.

4. Weerdesteyn V, de Niet M, van Duijnhoven HJ, Geurts AC. Falls in individuals with stroke. J Rehabil Res Dev. 2008;45(8):1195-1213.

197 5. Huang TT, Yang LH, Liu CY. Reducing the fear of falling among community-dwelling elderly adults through cognitive-behavioural strategies and intense Tai Chi exercise: a randomized controlled trial. J Adv Nurs. 2011;67(5):961-971.

200

6. Pang MY, Eng JJ. Fall-related self-efficacy, not balance and mobility performance, is related to accidental falls in chronic stroke survivors with low bone mineral density. Osteoporos Int. 2008;19(7):919-927.

203 7. Stevens JA, Corso PS, Finkelstein EA, Miller TR. The costs of fatal and nonfatal falls among older adults. Inj Prev. 2006;12:290-295.

205 8. Stevens JA, Mack KA, Paulozzi L, Ballesteros MF. Self-reported falls and fall-related injuries among persons aged>or=65 years--United States, 2006. J Safety Res. 
9. Schoene D, Wu SM, Mikolaizak AS, et al. Discriminative ability and predictive validity of the timed up and go test in identifying older people who fall: systematic review and meta-analysis. J Am Geriatr Soc. 2013;61(2):202-208.

10. Marschollek M, Rehwald A, Wolf KH, et al. Sensors vs. experts - a performance comparison of sensor-based fall risk assessment vs. conventional assessment in a sample of geriatric patients. BMC Med Inform Decis Mak. 2011;11:48.

11. Greene BR, Doheny EP, Walsh C, Cunningham C, Crosby L, Kenny RA. Evaluation of falls risk in community-dwelling older adults using body-worn sensors. Gerontology. 2012;58(5):472-480.

12. Gates S, Smith LA, Fisher JD, Lamb SE. Systematic review of accuracy of screening instruments for predicting fall risk among independently living older adults. J Rehabil Res Dev. 2008;45(8):1105-1116.

13. de Bruin ED, Najafi B, Murer K, Uebelhart D, Aminian K. Quantification of everyday motor function in a geriatric population. J Rehabil Res Dev. 2007;44(3):417-428.

14. Najafi B, Aminian K, Loew F, Blanc $Y$, Robert PA. Measurement of stand-sit and sit-stand transitions using a miniature gyroscope and its application in fall risk evaluation in the elderly. IEEE Trans Biomed Eng. 2002;49(8):843-851.

15. Najafi B, Aminian K, Paraschiv-lonescu A, Loew F, Bula CJ, Robert P. Ambulatory system for human motion analysis using a kinematic sensor: monitoring of daily physical activity in the elderly. IEEE Trans Biomed Eng. 2003;50(6):711-723.

16. Bowen DJ, Kreuter M, Spring B, et al. How we design feasibility studies. Am J Prev Med. 2009;36(5):452-457. 
230 17. Aminian K, Najafi B. Capturing human motion using body fixed sensors: outdoor measurement and clinical applications. Comp Anim Virtual Worlds. 2004;15:79-94.

18. Najafi B, Armstrong DG, Mohler J. Novel wearable technology for assessing spontaneous

19. Paraschiv-Ionescu A, Buchser EE, Rutschmann B, Najafi B, Aminian K. Ambulatory system

20. Chaudhuri S, Thompson H, Demiris G. Fall Detection Devices and Their Use With Older Adults: A Systematic Review. J Geriatr Phys Ther. 2014.

21. Schwickert L, Becker C, Lindemann U, et al. Fall detection with body-worn sensors : a systematic review. Z Gerontol Geriatr. 2013;46(8):706-719.

22. Igual R, Medrano C, Plaza I. Challenges, issues and trends in fall detection systems. Biomed Eng Online. 2013;12:66.

23. Bloch F, Gautier V, Noury N, et al. Evaluation under real-life conditions of a stand-alone fall detector for the elderly subjects. Ann Phys Rehabil Med. 2011;54(6):391-398.

24. English C, Manns PJ, Tucak C, Bernhardt J. Physical activity and sedentary behaviors in people with stroke living in the community: a systematic review. Phys Ther. 2014;94(2):185-196.

25. Rand D, Eng JJ, Tang PF, Jeng JS, Hung C. How active are people with stroke?: use of accelerometers to assess physical activity. Stroke. 2009;40(1):163-168. 
251 26. Sjoholm A, Skarin M, Churilov L, Nilsson M, Bernhardt J, Linden T. Sedentary behaviour and physical activity of people with stroke in rehabilitation hospitals. Stroke Res Treat. 2014;2014:591897.

254 27. Carroll DD, Courtney-Long EA, Stevens AC, et al. Vital signs: disability and physical activity--United States, 2009-2012. Morb Mortal Wkly Rep. 2014;63(18):407-413.

28. Banks G, Bernhardt J, Churilov L, Cumming TB. Exercise preferences are different after stroke. Stroke Res Treat. 2012;2012:890946.

29. Billinger SA, Arena R, Bernhardt J, et al. Physical activity and exercise recommendations for stroke survivors: a statement for healthcare professionals from the American Heart Association/American Stroke Association. Stroke. 2014;45(8):2532-2553.

30. Taylor-Piliae RE, Hoke TM, Hepworth JT, Latt LD, Najafi B, Coull BM. Effect of Tai Chi on physical function, fall rates and quality of life among older stroke survivors. Arch Phys Med Rehabil. 2014;95(5):816-824. 
267 Figure Legend

268 Figure 1: PAMSys ${ }^{\mathrm{TM}}$ Wearable Sensor Technology

269

270 Figure Caption

271 (A) PAMSys ${ }^{\mathrm{TM}}$ equipment (t-shirt and sensor).

272 (B) Summary of activities for a typical participant during 48 hours.

273 (C) Same data reported as the percentage of time (in minutes).

274 
275 Appendix: PAMSys ${ }^{\mathrm{TM}}$ Acceptability Questionnaire 
A

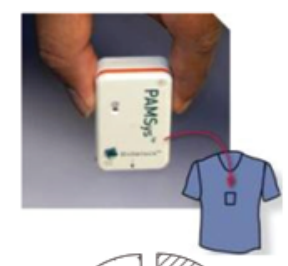

C

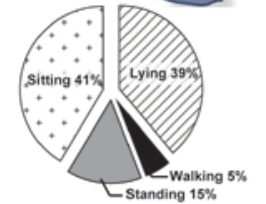

Walking: 140Min. (70 Min. per day) Standing: 419Min. (210 Min. per day) Sitting: $1203 \mathrm{Min}$. (601 Min. per day)
Lying: 1118Min. (559 Min. per day)
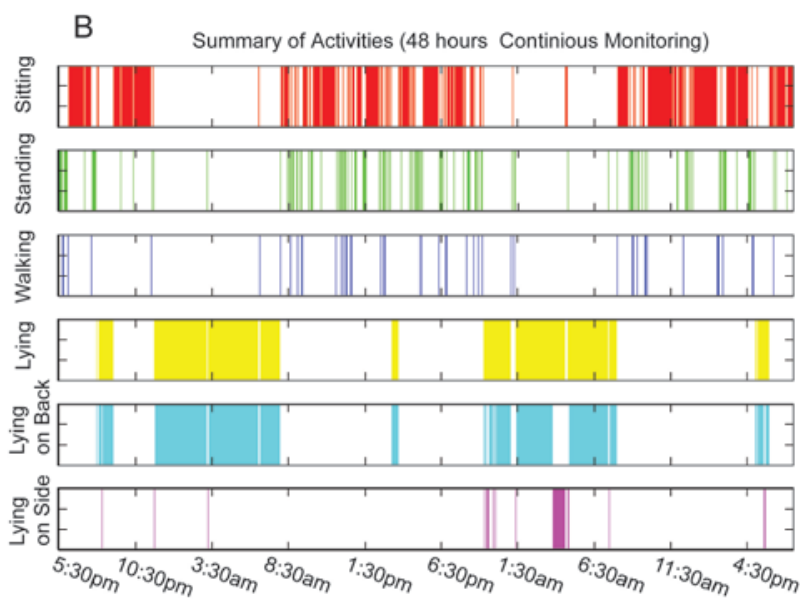

Time, $[\mathrm{Hr}]$
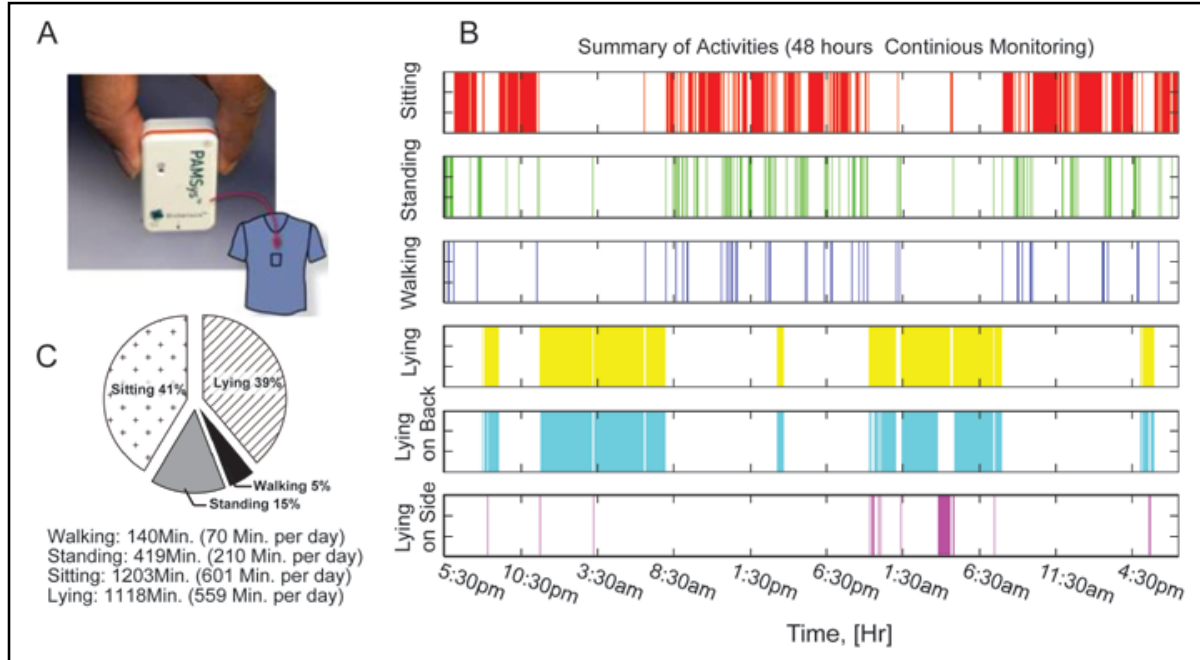
1 Table 1: Characteristics of Community-dwelling Stroke Survivors and Healthy Controls*

2

$$
\text { Stroke Survivors, } n=10 \quad \text { Controls, } n=10 \quad p \text {-value\# }
$$

Demographics

Age, mean $\pm S D$

$70 \pm 8$

$74 \pm 7$

0.216

Women, \%

70

89

0.228

Caucasian, \%

$60+$

78

0.258

Married, \%

50

College graduate, $\%$

70

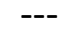

$---$

Retired, \%

80

Stroke-related Characteristics

Months post-stroke, mean \pm SD

$42 \pm 25$

$---$

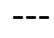

Ischemic stroke, \%

60

Hemorrhagic stroke, \%

40

Hemiparesis, \%

70

$--$

$---$

First stroke, \%

70

$--$

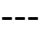

*self-reported, \# based on t-test or chi-square tests, + other ethnicities included African-American $(n=3$, $30 \%)$, and Mexican-American ( $n=1,10 \%)$ 
6 Table 2: PAMSys ${ }^{\mathrm{TM}}$ Acceptability among Community-dwelling Stroke Survivors

7

PAMSys $^{\mathrm{TM}}$ Equipment (i.e., t-shirt and sensor) $\quad n=10$

Comfort

Worn for the full 48 hours, \%

100

Comfortable to wear for 48 hours, $\%$

100

Willing to wear for another 48 hours, \%

100

Challenges

$\begin{array}{ll}\text { Interfered with normal everyday activities, \% } & 0\end{array}$

$\begin{array}{ll}\text { Trouble sleeping while wearing, \% } & 0\end{array}$

Difficulty removing to take shower/change clothes, $\% \quad 0$

$\begin{array}{ll}\text { Difficulty putting back on, \% } & 0\end{array}$

$\begin{array}{ll}\text { PAMSys }^{\mathrm{TM}} \text { t-shirt become wet/dirty, \% } & 0\end{array}$ 
9 Table 3: PAMSys ${ }^{\mathrm{TM}}$ Data Comparing Stroke Survivors with Age-Matched Controls

\begin{tabular}{|c|c|c|}
\hline \multirow[b]{3}{*}{ PAMSys $^{\mathrm{TM}}$ 48-hour Data } & \multicolumn{2}{|l|}{ Stroke } \\
\hline & Survivors, & Controls, \\
\hline & $\mathrm{n}=10$ & $\mathrm{n}=10$ \\
\hline \multicolumn{3}{|l|}{ Fall Risk } \\
\hline PT Duration (in seconds), mean \pm SD & $4.3 \pm 0.9 *$ & $3.6 \pm 0.3$ \\
\hline \multicolumn{3}{|l|}{ High Fall Risk $=>4$ seconds } \\
\hline Aborted PT Attempts (number per day), mean \pm SD & $11.5 \pm 4.9 *$ & 0 \\
\hline \multicolumn{3}{|l|}{ High Fall Risk= > 10 aborted attempts/day } \\
\hline \multicolumn{3}{|l|}{ Gait } \\
\hline Steps (number), mean \pm SD & $8,674 \pm 4,863^{*}$ & $12,600 \pm 2,937$ \\
\hline Speed (meters per second), mean \pm SD & $0.98 \pm 0.06$ & $1.0 \pm .19$ \\
\hline Duration ( $\%$ of total activity), mean \pm SD & $6.6 \pm 3.6^{*}$ & $9.4 \pm 1.8$ \\
\hline
\end{tabular}

PT=postural transition; *significantly worse, $\mathrm{p}<0.05$ 


\section{Appendix: PAMSys ${ }^{\mathrm{TM}}$ Acceptability Questionnaire}

1. Did you wear the PAMSys ${ }^{\mathrm{TM}}$ equipment (t-shirt and sensor) for the full 48 hours?

2. Was the PAMSys ${ }^{\mathrm{TM}}$ equipment (t-shirt and sensor) comfortable to wear for 48 hours?

3. Did you have trouble sleeping while wearing the PAMSys ${ }^{\mathrm{TM}}$ equipment ( $\mathrm{t}$-shirt and sensor)?

4. Did the PAMSys ${ }^{\mathrm{TM}}$ equipment interfere with your normal everyday activities?

5. Did the PAMSys ${ }^{\mathrm{TM}} \mathrm{t}$-shirt become dirty during this time?

6. Did the PAMSys ${ }^{\mathrm{TM}}$ t-shirt become wet during this time?

7. Did you use any body lotion while you were wearing the PAMSys ${ }^{\mathrm{TM}}$ equipment?

8. Did you remove the PAMSys ${ }^{\mathrm{TM}}$ equipment to take a shower/change clothes?

8.a. Did you have any difficulty removing the PAMSys ${ }^{\mathrm{TM}}$ equipment?

8.b. Did you have any difficulty putting the PAMSys ${ }^{\mathrm{TM}}$ equipment back on?

9. Did you return the t-shirt because it got dirty?

10. Did you return the t-shirt because it got wet?

11. Would you be willing to wear the PAMSys ${ }^{\mathrm{TM}}$ equipment (t-shirt and sensor) for another 48 hours?

12. Did you have any problems related to wearing the PAMSys ${ }^{\mathrm{TM}}$ equipment (t-shirt or sensor)?

If yes, please explain

13. What would you like to tell the next participant about what to expect while wearing the PAMSys ${ }^{\mathrm{TM}}$ equipment (t-shirt and sensor)?

Answer

14. Is there anything else you would like to tell us about your experience wearing the PAMSys ${ }^{\mathrm{TM}}$ equipment?

If yes, please explain 